\title{
Some Abelian, Tauberian and Core Theorems Related to the $(V, \lambda)$-Summability
}

\author{
Merve Temizer Ersoy ${ }^{1}$ \\ ${ }^{1}$ Department of Mathematics, Faculty of Science and Arts, Kahramanmaraş Sütcü İmam University, Kahramanmaraş, Turkey
}

\author{
Article Info \\ Keywords: A-statistically convergence, \\ core theorems, matrix transformations. \\ (Please, alphabetical order and at lease \\ one keyword) \\ 2010 AMS: 46A45, 40C05, $40 J 05$. \\ (Must be at least one and sequential) \\ Received: 5 April 2021 \\ Accepted: 4 June 2021 \\ Available online: 30 June 2021
}

\begin{abstract}
For a non-decreasing sequence of the positive integers tending to infinity $\lambda=\left(\lambda_{m}\right)$ such that $\lambda_{m+1}-\lambda_{m} \leq 1, \lambda_{1}=1 ;(V, \lambda)$-summability defined as the limit of the generalized de la Valée-Pousin of a sequence, [10]. In the present research, we establish some Tauberian, Abelian and Core theorems related to the $(V, \lambda)$-summability.
\end{abstract}

\section{Preliminaries}

Let $\mathbb{R}$ be the set of the reel numbers and $\mathbb{C}$ be the set of the complex numbers. Let $c$ and $\ell_{\infty}$ be the space of all complex valued convergent and bounded sequences, one by one. Let $\lambda=\left(\lambda_{m}\right)$ be a non-decreasing sequence of the positive integers tending to $\infty$ such that $\lambda_{1}=1, \lambda_{m+1} \leq \lambda_{m}+1$. A real number sequence $x=\left(x_{n}\right)$ is said to be $(V, \lambda)$-summable to the value $l$ if

$$
\lim _{m} t_{m}(x)=l
$$

exists, where

$$
t_{m}(x)=\frac{1}{\lambda_{m}} \sum_{n \in I_{m}} x_{n}, I_{m}=\left[m-\lambda_{m}+1, m\right] .
$$

By $(V, \lambda)$, we mean the set of all $(V, \lambda)$-summable sequences, i.e.,

$$
(V, \lambda)=\left\{x=\left(x_{n}\right): \lim _{m} t_{m}(x)=l \text { for some } l \in \mathbb{R}\right\} .
$$

Also, by $(V, \lambda)_{0}$ we denote the space of all sequences which $(V, \lambda)$-summable to zero. It is clear that in the case $\lambda_{m}=m$ for all $m$, $(V, \lambda)$-summability reduces to the Cesáro summability, [11]. If $x \in(V, \lambda)$ and $\lim _{m} t_{m}(x)=l$, then we have $(V, \lambda)-\lim x=l$.

Let $E$ be a subset of $\mathbb{N}$ (the set of natural numbers). Natural density $\delta$ of $E$ given by the following equality:

$$
\delta(E)=\lim _{n} \frac{1}{n}|\{k \leq n: k \in E\}| .
$$

The number sequence $x=\left(x_{k}\right)$ is said to be statistically convergent to the number $l$ if for every $\varepsilon>0, \delta\left(\left\{k:\left|x_{k}-l\right| \geq \varepsilon\right\}\right)=0$, [7]. In this case, we write: $s t-\lim x=l$, where $s t$ and $s t_{0}$ are the sets of all statistically convergent and statistically null sequences, respectively. For a given non-negative regular matrix $A$, the number

$$
\delta_{A}(K)=\lim _{n} \sum_{k \in K} a_{n k}
$$


is said to be the $A$-density of $K \subseteq \mathbb{N}$, [8]. A sequence $x=\left(x_{k}\right)$ is said to be $A$-statistically convergent to the number $s$ if for every $\varepsilon>0$, the set $\left\{k:\left|x_{k}-s\right| \geq \varepsilon\right\}$ has $A$-density zero, [2]. Thus, the following equation is valid: $s t_{A}-\lim x=s$. By $s t(A)$ and $s t(A)_{0}$, we respectively show the set of all $A$-statistically convergent and $A$-statistically null sequences.

For example, if we choose $E \subset \mathbb{N}$ such as $E=\left\{n^{2}: n=1,2,3 \cdots\right\}$ then it is easy to see that $\delta(E)=0$. A real number sequence $x=\left(x_{k}\right)$ is said to be statistically convergence to the number $l$ if for every $\varepsilon>0, \delta\left\{k:\left|x_{k}-l\right|\right\}=0$, [7]. For example, let

$$
x_{k}=\left\{\begin{array}{cc}
k & , \quad k=n^{2} \text { for all } n=1,2,3, \cdots \\
\frac{1}{k} & , \quad \text { otherwise. }
\end{array}\right.
$$

Then it obvious that $\lim x_{k}$ does not exist. But since $\delta(E)=\delta\left(\left\{n^{2}: n=1,2,3 \cdots\right\}\right)=0$, we write $s t-\lim x_{k}=\lim k \frac{1}{k}=0$. If $\left(x_{k}\right)$ is statistically convergence to a number $l$, then we write $s t-\lim x=l$. By $s t$ and $s t_{0}$, we denote the set of all statistically convergent and statistically null sequence, respectively. If a sequence is A-statistically convergent to $l$, then we can write $s t_{A}-\lim x=l$.

Let $x=\left(x_{k}\right)$ be a sequence in $\mathbb{C}$ and $R_{k}$ be the least convex closed region of complex plane containing $x_{k}, x_{k+1}, x_{k+2}, \ldots$. The Knopp Core (or $\mathscr{K}$-core) of $x$ is defined by the intersection of all $R_{k}(k=1,2, \ldots)$, [1, pp.137]. In [12], it is indicate that

$$
\mathscr{K}-\operatorname{core}(x)=\bigcap_{z \in \mathbb{C}} B_{x}(z)
$$

for any bounded sequence $x$, where $B_{x}(z)=\left\{w \in \mathbb{C}:|w-z| \leq \lim \sup _{k}\left|x_{k}-z\right|\right\}$.

In [6], the notion of the statistical core of a complex number sequence introduced by Fridy and Orhan [9] has been extended to the $A$-statistical core (or $s t_{A}$-core) and it is shown that for a $A$-statistically bounded seqeunce $x$

$$
s t_{A}-\operatorname{core}(x)=\bigcap_{z \in \mathbb{C}} C_{x}(z)
$$

where $C_{x}(z)=\left\{w \in \mathbb{C}:|w-z| \leq s t_{A}-\lim \sup \left|x_{k}-z\right|\right\}$. The inclusion theorems related to the $\mathscr{K}$-core and $s t_{A}$-core has been worked by many authors [3-5].

Let $D$ be an infinite matrix of complex entries $d_{n k}$ and $x=\left(x_{k}\right)$ be a complex valued sequence. Then $D x=\left\{(D x)_{n}\right\}$ is called the transformed sequence of $x$, if $(D x)_{n}=\sum_{k} d_{n k} x_{k}$ converges for each $n$. For two sequence spaces $M$ and $N$ we say that $D \in(M, N)$ if $D x \in N$ for each $x \in M$. If $M$ and $N$ are equipped with the limits $M-\lim$ and $N-\lim$, respectively, $D \in(M, N)$ and $N-\lim _{n}(D x)_{n}=M-\lim _{k} x_{k}$ for all $x \in M$, then we say $D$ regularly transforms $M$ into $N$ and write $D \in(M, N)_{\text {reg }}$.

Recently, similar works studied by some authors, see [13-17]. In the present paper, we have proved some Abelian, Tauberian and Core theorems related to the $(V, \lambda)$-summability.

\section{Tauberian and Abelian Theorems}

For any sequence spaces $X$ and $Y$, an Abelian theorem is a theorem such that states the inclusion $X \subset Y$. The Tauberian theorem is a one of the form $X \cap Z \subset Y$, where $Z$ is also a sequence space and $Y \subset X$.

Our first result for $(V, \lambda)$ is an Abelian theorem.

Theorem 2.1. $c_{(C, 1)} \subset(V, \lambda)$ if and only if

$$
\liminf _{m} \frac{m}{\lambda_{m}}=1
$$

where $c_{(C, 1)}$ is the space of all Cesáro summable sequences.

Proof. Let $x \in c_{(C, 1)}$ and

$$
\lim _{m} \frac{1}{m} \sum_{n=1}^{m} x_{n}=l
$$

Then, for any given $\varepsilon>0$ and enough large $m$,

$$
\left|\frac{1}{m} \sum_{n=1}^{m} x_{n}-l\right|<\varepsilon .
$$

Now, one can write that

$$
\begin{aligned}
\left|\frac{1}{\lambda_{m}} \sum_{n \in I_{m}}\left(x_{n}-l\right)\right| & =\left|\frac{1}{\lambda_{m}} \sum_{n=1}^{m}\left(x_{n}-l\right)-\frac{1}{\lambda_{m}} \sum_{n=1}^{m-\lambda_{m}}\left(x_{n}-l\right)\right| \\
& \leq \frac{m}{\lambda_{m}}\left|\frac{1}{m} \sum_{n=1}^{m}\left(x_{n}-l\right)\right|+\frac{m-\lambda_{m}}{\lambda_{m}}\left|\frac{1}{m-\lambda_{m}} \sum_{n=1}^{m-\lambda_{m}}\left(x_{n}-l\right)\right| \\
& \leq \frac{m}{\lambda_{m}} \varepsilon+\frac{m-\lambda_{m}}{\lambda_{m}} \varepsilon \\
& \leq \varepsilon\left(2 \frac{m}{\lambda_{m}}-1\right) .
\end{aligned}
$$

Therefore, it is clear that $\lim _{m} t_{m}(x)=l$ if and only if (2.1) holds. This completes the theorem. 
Since $c \subset c_{(C, 1)}$, the following result is obvious.

Corollary 2.2. If (2.1) holds then $c \subset(V, \lambda)$.

Theorem 2.3. $(V, \lambda)_{0} \cap c_{0} \subset\left(c_{0}\right)_{(C, 1)}$, where $\left(c_{0}\right)_{(C, 1)}$ is the space of all Cesáro summable to zero sequences.

Proof. Let $x \in(V, \lambda)_{0} \cap c_{0}$. Thus, for any $\varepsilon>0$ and enough large $m, n,\left|t_{m}(x)\right| \leq \varepsilon / 2$ and $\left|x_{n}\right| \leq \varepsilon / 2$. Hence, we have

$$
\begin{aligned}
\left|\frac{1}{m} \sum_{n=1}^{m} x_{n}\right| & =\left|\frac{1}{m} \sum_{n=1}^{m-\lambda_{m}} x_{n}+t_{m}(x)\right| \\
& \leq \frac{1}{m} \sum_{n=1}^{m-\lambda_{m}}\left|x_{n}\right|+\frac{\varepsilon}{2} \\
& \leq \frac{\varepsilon}{2}\left(1-\frac{\lambda_{m}}{m}+\frac{2}{m}\right) .
\end{aligned}
$$

Also, since $\lambda_{m} / m$ is bounded by 1 , the following inequality is true:

$$
\left|\frac{1}{m} \sum_{n=1}^{m} x_{n}\right| \leq \frac{\varepsilon}{m}
$$

which gives the result.

Since $\left(t_{m}(x)-l\right) \in(V, \lambda)_{0}$ and $\left(x_{n}-l\right) \in c_{0}$, we have the following outcome which is a Tauberian theorem.

Theorem 2.4. $(V, \lambda) \cap c \subset c_{(C, 1)}$.

\section{Core Theorems}

Definition 3.1. Let $R_{m}$ be the least closed convex hull containing $t_{m}, t_{m+1}, t_{m+2}, \ldots$. Then, $\mathscr{K}_{\lambda}$-core of $x$ is the intersection of all $R_{m}$, i.e.,

$$
\mathscr{K}_{\lambda}-\operatorname{core}(x)=\bigcap_{m=1}^{\infty} R_{m} .
$$

In fact, we define $\mathscr{K}_{\lambda}$-core of $x$ by the $\mathscr{K}$-core of the sequence $\left(t_{m}\right)$. Thus, one may state the following theorem which is an parallel of $\mathscr{K}$-core.

One can prove the following theorem by replacing $\left(t_{m}\right)$ in place of $\left(x_{k}\right)$, which is analogues of theorem given in [12] for Knopp core.

Theorem 3.2. Let, for any $z \in \mathbb{C}$,

$$
G_{x}(z)=\left\{w \in \mathbb{C}:|w-z| \leq \underset{m}{\limsup }\left|t_{m}(x)-z\right|\right\} .
$$

So, for any $x \in \ell_{\infty}$,

$$
\mathscr{K}_{\lambda}-\operatorname{core}(x)=\bigcap_{z \in \mathbb{C}} G_{x}(z)
$$

At present, we are in a position to construct the inclusion theorems. First of all, we prove several lemmas which will be helpful to the proof of the next theorems.

Lemma 3.3. Let $X$ be any sequence space. Then, $B \in(X,(V, \lambda))$ if and only if $D \in(X, c)$, where $D=\left(d_{n k}\right)$ is defined by

$$
d_{n k}=\left\{\frac{1}{\lambda_{n}} \sum_{j \in I_{n}} b_{j k},(n \in \mathbb{N})\right\} .
$$

Proof. Let $x \in X$ and take into consideration the equality

$$
\frac{1}{\lambda_{m}} \sum_{j \in I_{m}} \sum_{k=0}^{n} b_{j k} x_{k}=\sum_{k=0}^{n} \frac{1}{\lambda_{m}} \sum_{j \in I_{m}} b_{j k} x_{k} ;(m, n \in \mathbb{N})
$$

which yields as $n \longrightarrow \infty$ that

$$
\frac{1}{\lambda_{m}} \sum_{j \in I_{m}}(B x)_{j}=(D x)_{m} ;(m \in \mathbb{N}),
$$

where $D=\left(d_{n k}\right)$ is defined by (3.1).

Thus, it is obvious that $B \in(X,(V, \lambda))$ if and only if $D \in(X, c)$. As a result, the proof is complete.

For the special cases of the sequence space $X$, one can state the following lemmas. 
Lemma 3.4. $B \in(c,(V, \lambda))_{\text {reg }}$ if and only if

$$
\begin{aligned}
& \sup _{m} \sum_{k}\left|\frac{1}{\lambda_{m}} \sum_{n \in I_{m}} b_{n k}\right|<\infty, \\
& \lim _{m} \frac{1}{\lambda_{m}} \sum_{n \in I_{m}} b_{n k}=0, \forall k, \\
& \lim _{m} \sum_{k} \frac{1}{\lambda_{m}} \sum_{n \in I_{m}} b_{n k}=1 .
\end{aligned}
$$

Following lemma is an analogues of Theorem 3.2 in [4]. One can prove it by same technique. So, we omit the proof.

Lemma 3.5. $B \in\left(s t(A) \cap \ell_{\infty},(V, \lambda)\right)_{\text {reg }}$ if and only if $B \in(c,(V, \lambda))_{\text {reg }}$ and

$$
\lim _{m} \sum_{k \in E} \frac{1}{\lambda_{m}}\left|\sum_{n \in I_{m}} b_{n k}\right|=0
$$

for every $E \subset \mathbb{N}$ with $\delta_{A}(E)=0$.

By choosing $A$ as Cesáro matrix

$$
a_{n k}=\left\{\begin{array}{ccc}
1 / n & , & n \geq k \\
0 & , & \text { others }
\end{array}\right.
$$

we get following lemma.

Lemma 3.6. $B \in\left(S \cap \ell_{\infty},(V, \lambda)\right)_{\text {reg }}$ if and only if $B \in(c,(V, \lambda))_{\text {reg }}$ and

$$
\lim _{m} \sum_{k \in E} \frac{1}{\lambda_{m}}\left|\sum_{n \in I_{m}} b_{n k}\right|=0
$$

for every $E \subset \mathbb{N}$ with $\delta(E)=0$.

Now, we can give the following theorem.

Theorem 3.7. Let $\|B\|=\sup _{n} \sum_{k}\left|b_{n k}\right|<\infty$. Then, $\mathscr{K}_{\lambda}$-core $(B x) \subseteq \mathscr{K}$-core $(x)$ for all $x \in \ell_{\infty}$ if and only if $B \in(c,(V, \lambda))_{\text {reg }}$ and

$$
\lim _{m} \sum_{k} \frac{1}{\lambda_{m}}\left|\sum_{n \in I_{m}} b_{n k}\right|=1
$$

Proof. (Necessity). Let $x \in c$ with $\lim x=l$. Then, $\mathscr{K}$-core $(x)=\{l\}$ which implies that $\mathscr{K}_{\lambda}$-core $(B x) \subseteq\{l\}$. Since the assumption $\|B\|<\infty$ implies the boundedness of $B x, \mathscr{K}_{\lambda}$-core $(B x)=\{l\}$ and therefore $(V, \lambda)-\lim B x=l$. This implies that $B \in(c,(V, \lambda))_{\text {reg }}$.

Let's assume that the condition(3.3) is not satisfy. Then we have,

$$
\lim _{m} \sum_{k} \frac{1}{\lambda_{m}}\left|\sum_{n \in I_{m}} b_{n k}\right|>1
$$

The conditions of the Lemma 3.4 give us to choose two strictly increasing sequences $\left\{k\left(n_{i}\right)\right\}$ and $\left\{n_{i}\right\}(i=1,2, \ldots)$ of positive integers such that

$$
\sum_{k=0}^{k\left(n_{i}-1\right)} \frac{1}{\lambda_{m}}\left|\sum_{n \in I_{m}} b_{n_{i}, k}\right|<\frac{1}{4}, \sum_{k=k\left(n_{i-1}\right)+1}^{k\left(n_{i}\right)} \frac{1}{\lambda_{m}}\left|\sum_{n \in I_{m}} b_{n_{i}, k}\right|>1+\frac{1}{2}
$$

and

$$
\sum_{k=k\left(n_{i}\right)+1}^{\infty} \frac{1}{\lambda_{m}}\left|\sum_{n \in I_{m}} b_{n_{i}, k}\right|<\frac{1}{4}
$$

At present, let's define a sequence $x=\left(x_{k}\right)$ by

$$
x_{k}=\operatorname{sgn}\left(\frac{1}{\lambda_{m}} \sum_{n \in I_{m}} b_{n_{i}, k}\right), k\left(n_{i-1}\right)+1 \leq k<k\left(n_{i}\right),
$$

where $m$ is an integer as defined in the choosing $\lambda=\lambda_{m}$. Then, it is clear that $\mathscr{K}$-core $(x) \subseteq B_{x}(0)$. Also,

$$
\left|\sum_{k} \frac{1}{\lambda_{m}} \sum_{n \in I_{m}} b_{n_{i}, k} x_{k}\right| \geq \sum_{k=k\left(n_{i-1}\right)+1}^{k\left(n_{i}\right)} \frac{1}{\lambda_{m}}\left|\sum_{n \in I_{m}} b_{n_{i}, k}\right|-\sum_{k=0}^{k\left(n_{i}-1\right)} \frac{1}{\lambda_{m}}\left|\sum_{n \in I_{m}} b_{n_{i}, k}\right|-\sum_{k=k\left(n_{i}\right)+1}^{\infty} \frac{1}{\lambda_{m}}\left|\sum_{n \in I_{m}} b_{n_{i}, k}\right|>1+\frac{1}{2}-\frac{1}{4}-\frac{1}{4}=1 .
$$

Since $B \in(c,(V, \lambda))_{r e g}$, it follows that $(B x)$ has a subsequence whose $(V, \lambda)$-limit can not be in $B_{x}(0)$. This is a contradiction with that $\mathscr{K}_{\lambda}$-core $(B x) \subseteq \mathscr{K}$-core $(x)$. Hence, the condition (3.3) have to be satisfy. 
(Sufficiency). Let $w \in \mathscr{K}_{\lambda}$-core $(B x)$. So, for any given $z \in \mathbb{C}$, one get

$$
\begin{aligned}
|w-z| & \leq \limsup _{m}\left|t_{m}(B x)-z\right| \\
& =\limsup _{m}\left|z-\sum_{k} c_{m k} x_{k}\right| \\
& \leq \limsup _{m}\left|\sum_{k} c_{m k}\left(z-x_{k}\right)\right|+\underset{m}{\limsup }|z|\left|1-\sum_{k} c_{m k}\right| \\
& =\limsup _{m}\left|\sum_{k} c_{m k}\left(z-x_{k}\right)\right|
\end{aligned}
$$

where

$$
c_{m k}=\frac{1}{\lambda_{m}} \sum_{n \in I_{m}} b_{n k} .
$$

Now, let $\lim \sup _{k}\left|x_{k}-z\right|=l$. Subsequently, for any $\varepsilon>0,\left|x_{k}-z\right| \leq l+\varepsilon$ whenever $k \geq k_{0}$. Thus, the following inequality applies:

$$
\begin{aligned}
\left|\sum_{k} c_{m k}\left(z-x_{k}\right)\right| & =\left|\sum_{k<k_{0}} c_{m k}\left(z-x_{k}\right)+\sum_{k \geq k_{0}} c_{m k}\left(z-x_{k}\right)\right| \\
& \leq \sup _{k}\left|z-x_{k}\right| \sum_{k<k_{0}}\left|c_{m k}\right|+(l+\varepsilon) \sum_{k \geq k_{0}}\left|c_{m k}\right| \\
& \leq \sup _{k}\left|z-x_{k}\right| \sum_{k<k_{0}}\left|c_{m k}\right|+(l+\varepsilon) \sum_{k}\left|c_{m k}\right| .
\end{aligned}
$$

Therefore, applying $\limsup _{m}$ and combining (3.4) with (3.5), we have

$$
|w-z| \leq \limsup _{m}\left|\sum_{k} c_{m k}\left(z-x_{k}\right)\right| \leq l
$$

which shows that $w \in \mathscr{K}$-core $(x)$. The proof is completed.

Theorem 3.8. Let $\|B\|=\sup _{n} \sum_{k}\left|b_{n k}\right|<\infty$. Then, $\mathscr{K}_{\lambda}$-core $(B x) \subseteq s t_{A}$-core $(x)$ for all $x \in \ell_{\infty}$ if and only if $B \in\left(\text { st }(A) \cap \ell_{\infty},(V, \lambda)\right)_{\text {reg }}$ and the condition (3.3) are satisfy.

Proof. (Necessity). By choosing $x \in s t(A) \cap \ell_{\infty}$, as in Theorem 3.7, we immediately have that $B \in\left(s t(A) \cap \ell_{\infty},(V, \lambda)\right)_{\text {reg. }}$.

On the other hand, since $s t_{A}$-core $(x) \subseteq \mathscr{K}$-core $(x)$ for any sequence $x$ [6], the necessity of the condition (3.3) follows from Theorem 3.7.

(Sufficiency). Let we take $w \in \mathscr{K}_{\lambda}$-core $(B x)$. So, we have again the condition (3.4). At present, if $s t_{A}-\lim \sup \left|x_{k}-z\right|=s$, then for any $\varepsilon>0$, the set $E$ defined by $E=\left\{k:\left|x_{k}-z\right|>s+\varepsilon\right\}$ has zero $A$-density. At present, we get

$$
\begin{aligned}
\left|\sum_{k} c_{m k}\left(z-x_{k}\right)\right| & =\left|\sum_{k \in E} c_{m k}\left(z-x_{k}\right)+\sum_{k \notin E} c_{m k}\left(z-x_{k}\right)\right| \\
& \leq \sup _{k}\left|z-x_{k}\right| \sum_{k \in E}\left|c_{m k}\right|+(s+\varepsilon) \sum_{k \notin E}\left|c_{m k}\right| \\
& \leq \sup _{k}\left|z-x_{k}\right| \sum_{k \in E}\left|c_{m k}\right|+(s+\varepsilon) \sum_{k}\left|c_{m k}\right| .
\end{aligned}
$$

Hence, applying the operator $\limsup _{m}$ and using the condition (3.3) with (3.2), we can write that

$$
\underset{m}{\limsup }\left|\sum_{k} c_{m k}\left(z-x_{k}\right)\right| \leq s+\varepsilon
$$

Finally, combining (3.4) with (3.6), we get

$$
|w-z| \leq s t_{A}-\underset{k}{\limsup }\left|x_{k}-z\right|
$$

which shows that $w \in s t_{A}$-core $(x)$.

As a consequence of Theorem 3.8, we get

Corollary 3.9. Let $\|B\|=\sup _{n} \sum_{k}\left|b_{n k}\right|<\infty$. Then, $\mathscr{K}_{\lambda}$-core $(B x) \subseteq$ st-core $(x)$ for all $x \in \ell_{\infty}$ if and only if $B \in\left(\text { st } \cap \ell_{\infty},(V, \lambda)\right)_{\text {reg }}$ and $(3.3)$ holds.

\section{Conclusion}

In this paper, we obtained new some Tauberian, Abelian and Core theorems related to the $(V, \lambda)$-summability.

\section{Acknowledgements}

The authors would like to express their sincere thanks to the editor and the anonymous reviewers for their helpful comments and suggestions. 


\section{Funding}

There is no funding for this work.

\section{Availability of data and materials}

Not applicable.

\section{Competing interests}

The authors declare that they have no competing interests.

\section{Author's contributions}

All authors contributed equally to the writing of this paper. All authors read and approved the final manuscript.

\section{References}

[1] R. G. Cooke, Infinite Matrices and Sequence Spaces, Macmillan, New York, 1950.

[2] J. Connor, On strong matrix summability with respect to a modulus and statistical convergence, Canad. Math. Bul. 32(1989), 194-198.

[3] H. Çoşkun, C. Çakan, Infinite matrices and $\sigma$-core, Demonstratio Math., 34(2001), 825-830.

[4] H. Çoşkun, C. Çakan, On some new inequalities related to the $F_{\mathscr{B}}$-convergence, Tamsui Oxford J. Math. Sci., 19(2)(2003), 131-140.

[5] H. Çoskun, C. Çakan, A class of matrices mapping $\sigma$-core into A-statistical core, Tamsui Oxford Journal of Math. Sci., 20(1) (2004) 17-25.

[6] K. Demirci, A-statistical core of a sequence, Demonstratio Math., 160(2000), 43-51.

[7] H. Fast, Sur la convergence statisque, Colloq. Math., 2(1951), 241-244.

[8] A. R. Freedman, J. J. Sember, Densities and summability, Pasific J. Math., 95(1981), 293-305.

[9] J. A. Fridy, C. Orhan, Statistical core theorems, J. Math., Anal. Appl. 208(1997), 520-527.

[10] L. Leindler, Uber die de la Vallée-Pousinsche Summierbarkeit allgemeiner Orthogonalreihen, Acta Math. Acad. Sci. Hungar., $16(1965), 375-387$.

[11] I. J. Maddox, Elements of Functional Analysis, Cambridge University Press, Cambridge 1970.

[12] A. A. Shcherbakov, Kernels of sequences of complex numbers and their regular transformations, Math. Notes, 22(1977), 948-953.

[13] U. Ulusu, E. Dündar, Asymptotically I-Cesaro equivalence of sequences of sets, Universal Journal of Mathematics and Applications, 1(2) (2018), 101-105.

[14] R. Kama, Spaces of vector sequence defined by the f-statistical convergence and some characterizations of normed spaces, Revista de la Real Academia de Ciencias Exactas, 4(2) (2020), 1-9.

[15] C. Unal, Ergodic Theorem in Grand Variable Exponent Lebesgue Spaces, Mathematical Science and Applications E-notes, 8(2) (2020), $130-134$.

[16] O. Talo, E. Yavuz, H. Çoşkun, Tauberian Theorems for Statistical Logarithmic Summability of Strongly Measurable Fuzzy Valued Functions, Communications in Advanced Mathematical Sciences, 2 (2020), 91-100.

[17] V. Renukadevi, S. Vadakasi, On Various g-Topology in Statistical Metric Spaces, Universal Journal of Mathematics and Applications, 2(3) (2019), 107-115. 